Risk Analysis and Risk Management

\title{
The Statistical Face of a Region under Monsoon Rainfall in Eastern India
}

\author{
Subrata Kundu ${ }^{1}$, Kaushik Jana ${ }^{2}$, Debasis Sengupta ${ }^{3}$ \\ ${ }^{1}$ The George Washington University, USA \\ ${ }^{2}$ Imperial College, UK \\ ${ }^{3}$ Indian Statistical Institute, Kolkata, India
}

doi: https://doi.org/10.21467/abstracts.93.11

\begin{abstract}
A region under rainfall is a contiguous spatial area receiving positive precipitation at a particular time. The probabilistic behavior of such a region is an issue of interest in environmental and meteorological studies. A region under rainfall can be viewed as a shape object of a special kind, where scale and rotational invariance are not necessarily desirable attributes of a mathematical representation.

For modeling variation in objects of this type, we propose an approximation of the boundary that can be represented as a real valued function, and arrive at further approximation through functional principal component analysis, after suitable adjustment for asymmetry and incompleteness in the data. The analysis of an open access satellite data set on monsoon precipitation over Eastern India leads to explanation of most of the variation in shapes of the regions under rainfall through a handful of interpretable functions that can be further approximated parametrically. The most important aspect of shape is found to be the size followed by contraction/elongation, mostly along two pairs of orthogonal axes. The different modes of variation are remarkably stable across calendar years and across different thresholds for minimum size of the region.
\end{abstract}

